\title{
Benzalkonium chloride (BAK) induces apoptosis or necrosis, but has no major influence on the cell cycle of Jurkat cells
}

\author{
Dorota Pozarowska ${ }^{1 *}$, Piotr Pozarowski ${ }^{*}$ \\ ${ }^{1}$ Department of Ophthalmology, Medical University of Lublin, Poland \\ ${ }^{2}$ Department of Clinical Immunology, Medical University of Lublin, Poland \\ *Equal contribution of both authors
}

\begin{abstract}
Benzalkonium chloride (BAK) is a cationic detergent with a very slow turnover. Because of its strong antibacterial activities, BAK is widely used especially in dentistry and ophthalmology. It is the most commonly used preservative in topical ophthalmic medications. Due to chronicity and widespread use of such treatments, BAK's side effects are of great importance. BAK toxicity for adherent cells, probably related to its pro-oxidative activities, is time- and dose-dependent. Although lymphocytes often infiltrate superficial eye tissues, the BAK influence on them is yet to be established. The aim of this study was to check BAK cytotoxicity on T lymphocytic Jurkat line cells and to verify the suggestion that BAK can induce G2M cell blocks. A dose- and time-dependent cytotoxic effect of BAK on lymphoid cells in relatively low concentrations was shown in this study. In lower concentrations, it shows a moderate apoptotic and minimal antiproliferative effect on Jurkat cells, while in higher concentrations it shows a rapid necrotic effect. No G2M cell blocks were observed. Our findings could suggest lymphoid dysfunction during intensive, prolonged topical BAK treatment, even at dosages relatively non-toxic to epithelial eye cells. (Folia Histochemica et Cytobiologica 2011; Vol. 49, No. 2, pp. 225-230)
\end{abstract}

Key words: BAC, toxicity, apoptosis, necrosis, viability, cell cycle, proliferation, cornea, conjunctiva, in vitro

\section{Introduction}

Benzalkonium chloride (BAK) is a cationic detergent expressing a high affinity to membrane proteins. It changes the ionic resistance of the cell membranes [1]. BAK, due to its antibacterial activities, is widely used especially in ophthalmology and dentistry. Its concentration in oral disinfectant mouthwash can reach as much as $10 \%$ [2] whereas in eye drops it varies between $0.004-0.05 \%$ [3]. Due to chronicity and widespread use of such treatments, BAK's side effects are of great importance. BAK's toxicity, probably related to its pro-oxidative activities [2], is time-

\footnotetext{
Correspondence address: D. Pozarowska,

Department of Ophthalmology, Medical University,

Chmielna Str. 1, 20-079 Lublin, Poland;

tel./fax: (+ 48 18) 53261 49;

e-mail:ppozarowski@yahoo.com
}

and dose-dependent. It also causes protein denaturation and disruption of the cytoplasmatic membranes [1]. This phenomenon could be helpful in increasing the penetrative abilities of the ophthalmologic solution's active compounds [4,5]. Moreover, some of the active ophthalmological compounds e.g. prostaglandins exert a protective effect against the cytotoxicity of BAK [1, 6-8]. BAK can also decrease expression of MUC5AC on conjunctival cells, causing mucin deficiency and dry eyes in patients undergoing prolonged topical ophthalmic therapy $[1,6]$ and influence the generation of inflammation. Conjunctival expression of intercellular adhesion molecule-1 (ICAM-1, CD54), platelet-endothelial cell adhesion molecule-1 (PECAM-1, CD31) and HLA-DR could be increased after their contact with BAK attracting and activating lymphocytes $[1,7]$.

In our recent publication, we showed that eight different commercially available anti-glaucoma topi- 
cal medications induce cell necrosis or apoptosis and suppress proliferation of human corneal line cells in a dose- and time-dependent manner. Moreover, some of them cause G2M cell blocks. All these ophthalmologic drops contain BAK as a preservative [9]. The aim of this paper was to establish the role of BAK in these observed phenomena.

\section{Material and methods}

Cell culture. A Jurkat cell line purchased from American Tissue and Cell Collection (Rockville, MD, USA) was used in all experiments. Jurkat is a pseudodiploid human cell line originated from acute T-cell leukemia. Cells were cultured at $37^{\circ} \mathrm{C}$ in a humidified atmosphere with the addition of $5 \%$ $\mathrm{CO}_{2}$ in air in RPMI 1640 (Biochrom AG, Berlin, Germany) supplemented with $10 \%$ newborn calf serum (Gibco, Paisley, UK), $100 \mathrm{U} / \mathrm{ml}$ penicillin (Sigma, Poznan, Poland), 200 $\mu \mathrm{g} / \mathrm{ml}$ neomycin (Sigma) and $100 \mu \mathrm{g} / \mathrm{ml}$ streptomycin (Sigma). The cultures were periodically checked for Mycoplasma infections. In order to maintain asynchronous, exponential growth, cultures were splitted daily by $1: 1$ dilution.

BAK experiments. Benzalkonium chloride (BAK) was purchased from Sigma. 5\% ( 140 mM) stock solution of BAK was prepared in distilled water and stored in a refrigerator for a couple of days. BAK stock solution was administered directly into the culture flasks to obtain the final concentration, as indicated in the text and in the figure captions. BAK's influence on cells was checked in the range between $0.000001-0.5 \%$ (28 $\mathrm{nM}-14 \mathrm{mM})$. In key concentrations, experiments were performed in at least five repetitions.

Viability experiments. Propidium iodide (PI) (Sigma) was added to the cell suspension collected directly from the culture flask at a final concentration of $1 \mu \mathrm{g} / \mathrm{ml}$. After a short incubation, the cells were analyzed in a flow cytometer (FACSCalibur, BD Biosciences) equipped with a $488 \mathrm{~nm}$ laser. Specific PI fluorescence was measured as FL3. FCS low $/ \mathrm{PI}^{+}$cells were considered as dead, and FCS high/PI- cells as live.

Cell cycle experiments. The cells, treated with different concentrations of medications for various time intervals as indicated in the 'Results' section, were stained with acridin orange (AO) and subsequently analyzed by flow cytometry, as described previously $[10,11]$. Using acridin orange assay enabled us to measure both RNA and DNA content in a single cell. This is crucial, not only for cell cycle positioning, but also to distinguish between apoptosis and necrosis (Figure 1). At least 10,000 cells were analyzed [10-12].

Statistical analysis. GraphPad Prism software and Wilcoxon matched paired tests were applied for statistical analysis as well as for preparation of distribution diagrams.

\section{Results}

BAK's dose- and time-dependent toxic effects were investigated in this study. In BAK concentrations higher than $0.0001 \%$, Jurkat cells rapidly die showing a necrotic mode of cell death (Figure 2). Using BAK in the range between 0.00001-0.0001\% (0.28$-2.8 \mu \mathrm{M})$ slightly induces apoptosis in a dose- and timedependent mode (Figures 1, 3). Jurkat cell median apoptosis after 48 hours of cultivation significantly increased from $8.3 \%$ seen in control to $17 \%$ in $0.0001 \%(2.8 \mu \mathrm{M})$ BAK concentration. BAK does not induce any type of cell death in concentrations lower than $0.00001 \%(0.28 \mu \mathrm{M})$ (data not shown).

These results were confirmed by viability results. BAK showed dose- and time-dependent effects on the viability of Jurkat cells with almost no effect in concentrations lower than $0.00001 \%(0.28 \mu \mathrm{M})$ (data not shown) and a slight effect in the range between $0.00001-0.0001 \%(0.28-2.8 \mu \mathrm{M})$. Dramatic loss of viability was observed in concentrations higher than $0.0001 \%(2.8 \mu \mathrm{M})$ (Figure 2).

No major cell cycle blocks as a result of BAK influence were seen in concentrations up to $0.0001 \%$ $(2.8 \mu \mathrm{M})$ (Figure 3$)$. A slight but significant decrease of cell numbers in $\mathrm{S}$ phase seen in cultures with addition of $0.00003-0.0001 \%(1.4-2.8 \mu \mathrm{M}) \mathrm{BAK}$, especially after 48 hours of cultivation, was observed. This phenomenon could be the result of BAK's cytostatic effect (Figure 3). Due to rapid cell death, cell cycle analysis could not be performed in higher BAK concentrations.

\section{Discussion}

Eye preservative turnover is very slow. The lipophilic nature of most of them causes them to bind to the ocular tissues immediately after application and prolongs their action for as long as hundreds of hours. The half-time of BAK elimination from the eye surface is approximately 20 hours [13, 14]. This is why we decided to use the long term culture model in this study. We showed that BAK induces necrosis (concentration $>0.0001 \%$ ), apoptosis (concentration $0.00001-0.0001 \%$ ) or has no major influence (concentration $<0.00001 \%$ ) on Jurkat line cells. The Jurkat cell line originates from T lymphocytes. Although lymphocytes do not infiltrate the cornea in physiological conditions, they are present in other parts of the anterior segment of the eye as an anterior chamber or a conjunctiva in both physiological and pathological conditions and are a subject for BAK stimulation. Moreover, exposure to BAK increases permability of eye tissues $[4,5]$ and can have a proinflammatory effect. It increases corneal epithelial cell expression of cell 

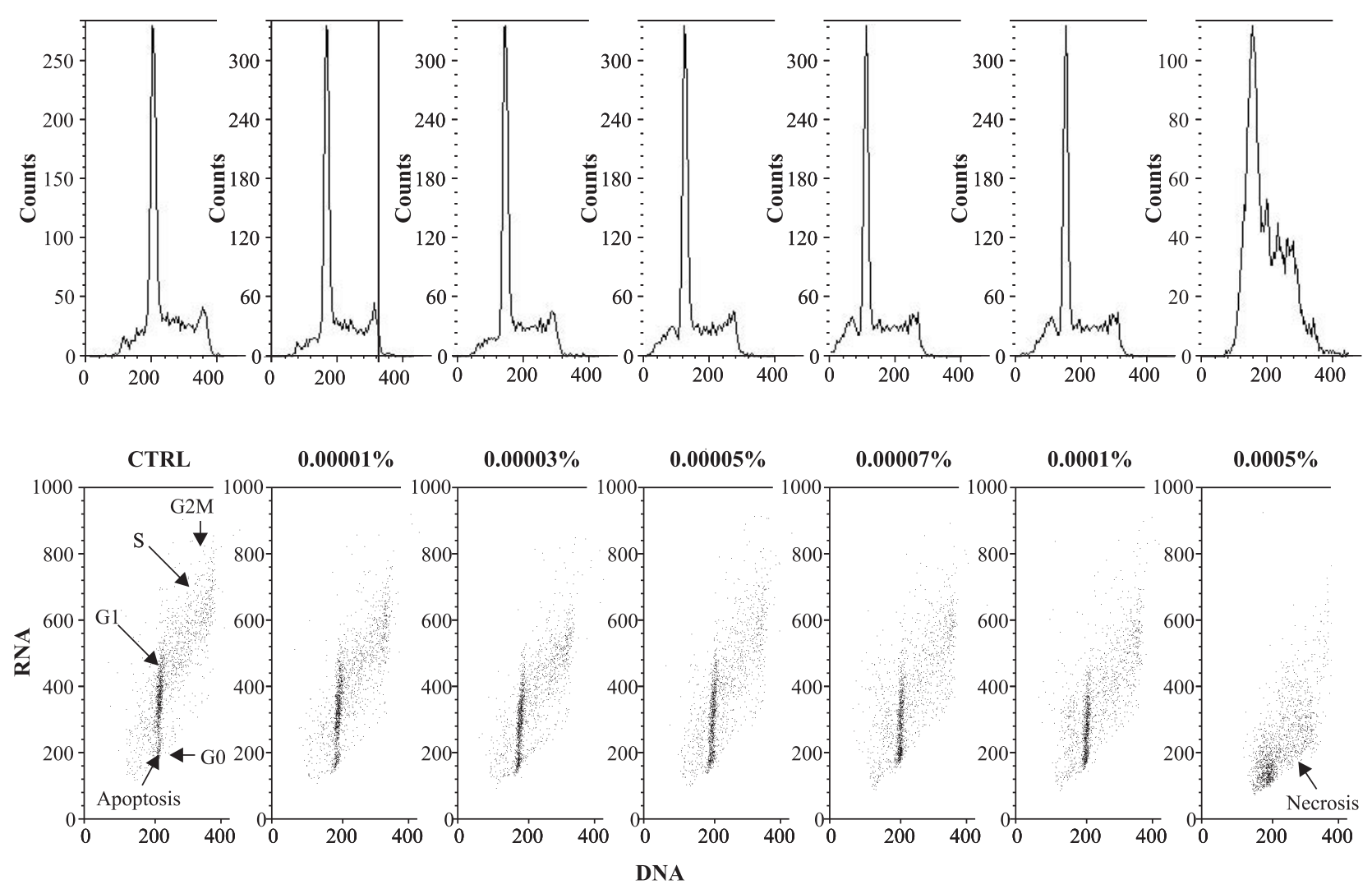

Figure 1. After 48 hours' cultivation of Jurkat cells in the absence or presence of benzalkonium chloride (BAK) at different concentrations, cells were stained with acridine orange (AO) and subsequently analyzed in flow cytometry (FACS Calibur, BD Biosciences). Usage of AO assay enabled us to measure both DNA and RNA cell contents. DNA histograms are shown in upper row. Cell cycle distribution is also presented as dotplots in lower row. Please note that necrotic cells show not only lower RNA, but also DNA content

adhesion molecules helping lymphocytes to reach the site of inflammation, and also expression of HLA-DR, which is crucial for antigen presentation needed for lymphocyte activation [1,7]. This data is supported by clinical observations showing an immunologic reaction with increased presence of lymphocytes, Langerhans cells and macrophages in patients subjected to chronic application of preservatives. Moreover, sometimes such inflammations can lead to subconjunctival fibrosis [15].

In our recent study, we showed that almost all examined commercially available anti-glaucoma medications decrease the number of corneal epithelial cells during their cultivation due to induction cell death and proliferation suppression [9]. This observation can be explained not only by the effect of active substances, but also by BAK action. Many other in vitro studies have shown that preservatives are cytotoxic, especially for the cells of the ocular surface: conjunctival and corneal epithelium and keratocytes $[7,9,14$, 16-19] but also for the deeper ocular tissues (fibroblast of Tenon's capsule, cells of lens epithelium and trabecular meshwork) $[15,20]$. Although the cytotoxic effect of BAK is well documented in patients [15] and on cultivated adherent cells, its influence on lymphocytes seems not to have been explored until this study. Lymphocytes seem to be very sensitive to the cytotoxic effect of BAK. Cytotoxic and proapoptotic BAK concentrations for corneal and conjunctiva cells are higher than seen in this study for lymphocytes, and vary between 0.0001 and $0.02 \%$, but most of them are close to the latter figure [5, 7, 8, 14, 17, 18]. Huge differences in these concentrations are probably due to differences in methods of cell treatments by BAK (especially in treatment and recovery time) and sensitivities of assays. Trabecular meshwork cells also seem to be very sensitive to BAK stimulation: in concentrations as low as $0.00015 \%$, it kills about $20 \%$ of cells [20].

Our present data also shows minimal anti-proliferative BAK effect on lymphoid cells. The number of cells in S phase was slightly reduced in cultures (especially those lasting 48 hours) treated with a subtoxic dosage of BAC. This suggests that in clinical conditions, the proliferation of lymphocytes is more resistant for BAK suppressive influence than cells 

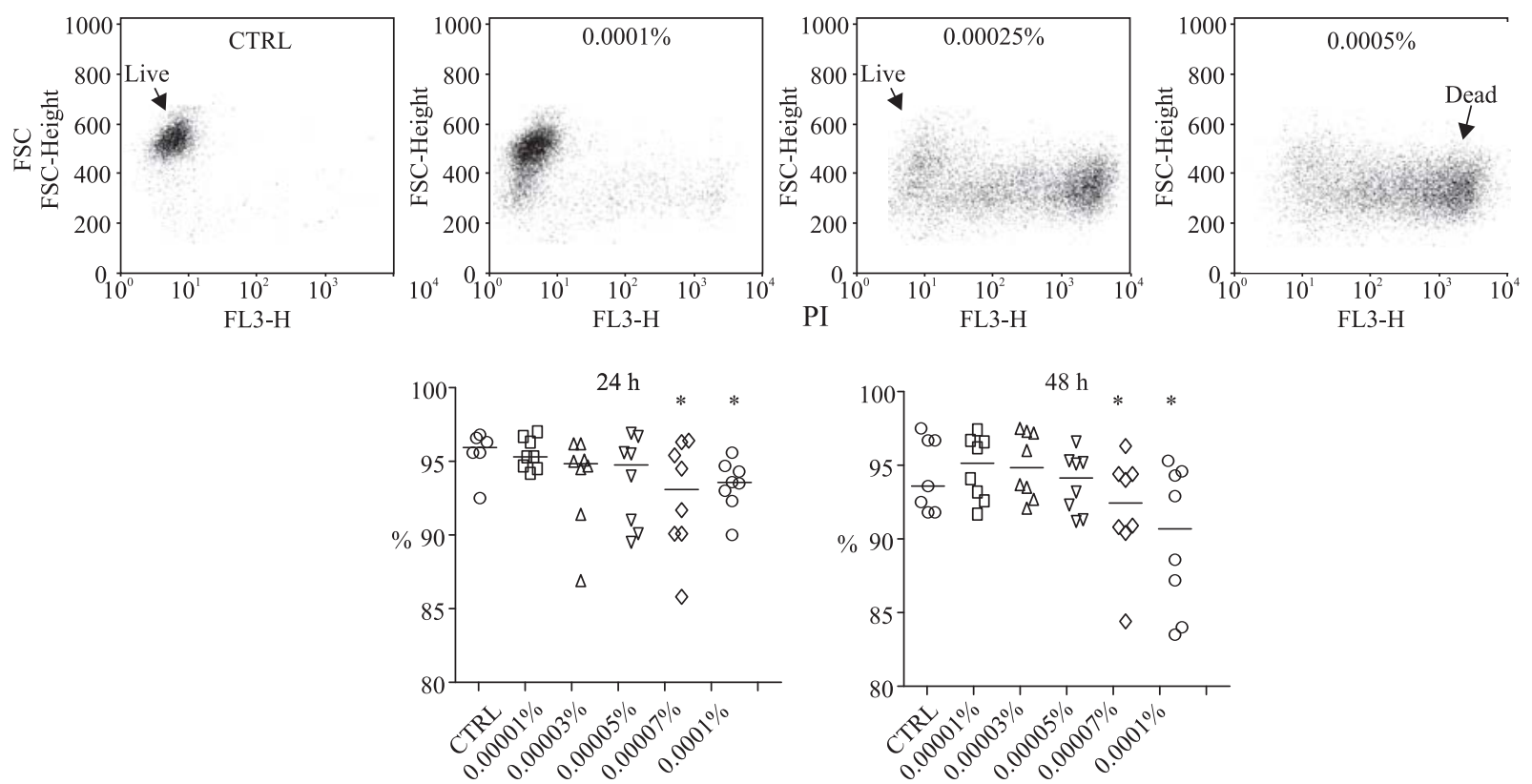

Figure 2. Jurkat cells were cultured in the absence or presence of different concentrations of benzalkonium chloride (BAK) for 24 or 48 hours. To check their viability, cells were stained with PI $(1 \mu \mathrm{g} / \mathrm{ml})$ and subsequently analyzed in a flow cytometer (FACSCalibur). In upper row, dotplots show cellular size (FSC) versus fluorescence of PI (each dot represents a single cell) of Jurkat cells cultured for 24 hours. Dead cells were defined as FCS low/PI+ and live ones as FCS high/PICalculated numbers of live cells are presented in lower panel. Medians are marked as lines. Statistical analysis and graphs were performed using GraphPad Prism software. Significant differences $(\mathrm{p}<0.05)$ to the control are marked as *
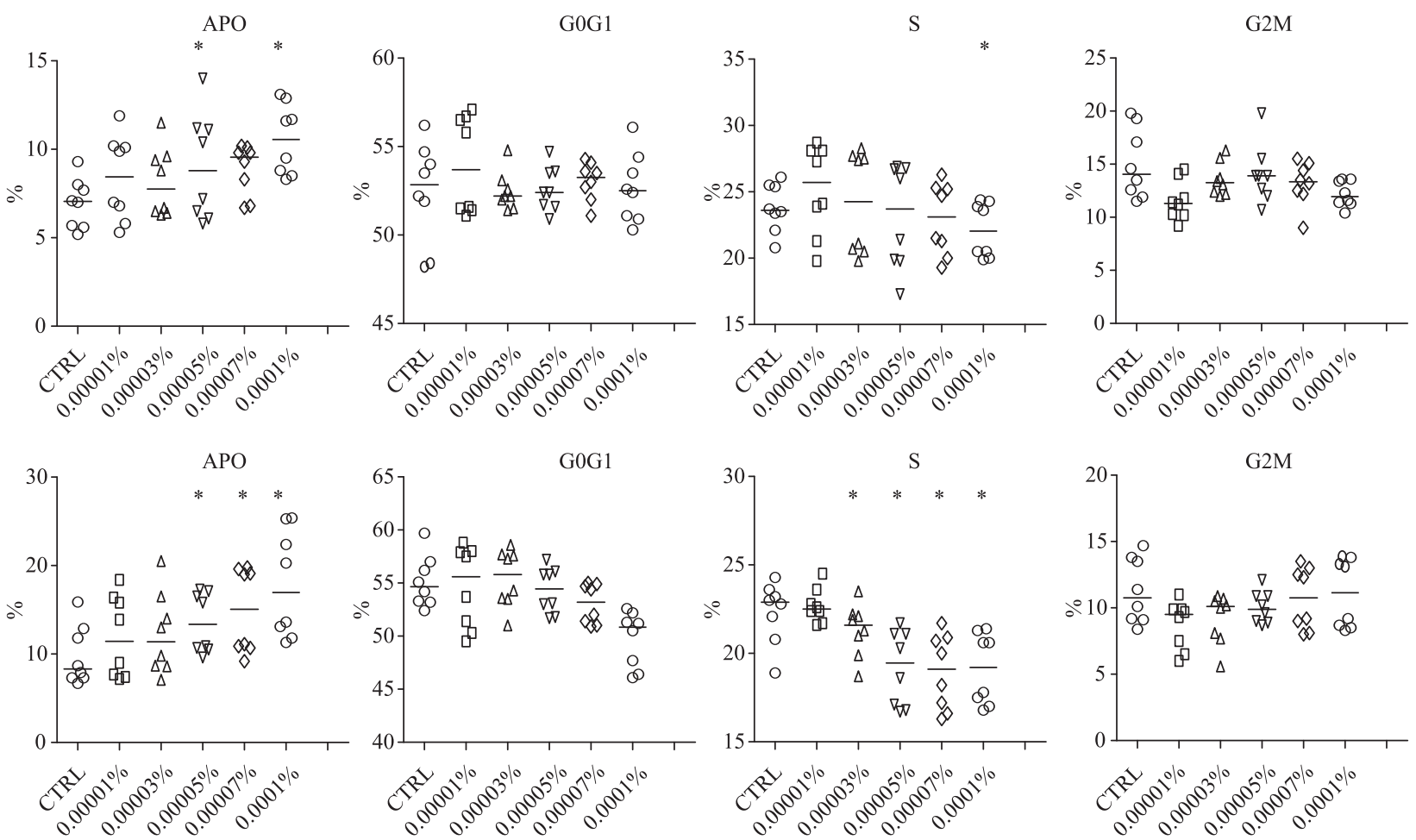

Figure 3. Jurkat cells were cultured in the absence or presence of benzalkonium chloride (BAK) at different concentrations for 24 or 48 hours, stained with acridine orange (AO) as described previously [10, 11] and subsequently analyzed via flow cytometry (FACSCalibur). Cell cycle distributions were calculated and are presented as distribution diagrams. Medians are marked as lines. Diagrams were prepared using GraphPad Prism software. Significant differences $(p<0.05)$ to the control are marked as * 
originating from the eye. On the other hand, it should be stated that this anti-proliferative lymphocytic dosage is still much lower than that for epithelial cells. Sub-toxic BAK stimulation was also applied on primary human gingival fibroblasts. An IC50 dose of BAK was unable to break down artificial G0/G1 block, resulting in no S and G2M cell progression [2].

Pauly et al. reported that $0.01 \%$ BAK stimulation of corneal epithelial cells tripled their proliferation (measured as percentage of $\mathrm{Ki}-67^{+}$cells) as a mechanism of cell defense. On the other hand, lower BAK concentrations do not alter the proliferative abilities of cells, while higher concentrations (even $0.02 \%$ ) completely abolish proliferation [5]. Proliferation of human corneal keratocytes has also been measured by $\left[{ }^{3} \mathrm{H}\right]$ thymidine methodology. BAK at a concentration of $0.01 \mathrm{mg} / \mathrm{ml}$ significantly decreases the proliferation of such cells, whereas its lower concentration does not affect it [21]. It also should be stated that BAK influences superficial cells, e.g. corneal epithelial cells or conjunctival cells, in higher concentrations than on infiltrating lymphocytes, even including BAK's effect on increasing the permeability of eye tissue.

Our findings in this study do not confirm the suggestion that BAK can induce G2M block. Such blocks were observed in some human corneal line cells treated with commercial anti-glaucoma medication containing BAK [9]. This observation has not been confirmed in vitro by others on corneal cells [18], primary gingival fibroblasts [2] or on lymphoid line cells in this study. Most likely, the G2M blocks observed in our previous publication were due to active compounds of ophthalmic medication and not due to BAK's influence, but studies on corneal epithelial cells still need to be performed. BAK does not induce G2M block enabling cell cycle progression and thus lymphocytes can proliferate and respond properly for antigen stimulation. It should be state however that Jurkat cells are neoplastic ones and they differ from normal ones.

In conclusion, we want to state that BAK is relatively toxic to lymphoid cells, especially causing necrosis. In lower concentrations, it also shows moderate apoptotic and minimal antiproliferative effect on such cells. Other eye cells, especially superficial ones such as corneal epithelial cells and conjunctival cells, seem to be more resistant to BAK treatment. This observation could suggest possible lymphoid dysfunction during intensive, prolonged topical BAK treatment, even at a dosage relatively non-toxic to epithelial eye cells.

\section{References}

1. Pozarowska D. Toxic effects of antiglaucoma drugs on superficial tissues of the eye. Int Rev Allergol Clin Immunol. 2008:14:110-114.

2. Nomura Y, Bhawal UK, Nishikioro R, Sawajiri M, Maeda T, Okazaki M. Effect of high-dose major components in oral disinfectants on the cell cycle and apoptosis in primate human gingival fibroblasts in vitro. Dent Material J. 2010;29:75-83.

3. Yee RW. The effect of drop vehicle on the efficacy and side effects of topical glaucoma therapy: a review. Curr Opin Ophtalmol. 2007;18:134-139.

4. Yi X, Wang T, Yu FS. Corneal epithelial tight junctions and their response to lipopolysaccharide challenge. Inv Ophthalmol Vis Sci. 2000;41:4093-4100.

5. Pauly A, Meloni M, Brignole-Baudouin F, Warnet J-M, Baudouin Ch. Multiple endpoint analysis of the 3D-reconstituted corneal epithelium after treatment with Benzalkonium chloride: early detection of toxic damage. Inv Ophthalmol Vis Sci. 2009;50:1644-1652.

6. Pisella PJ, Debbasch C, Hamard P et al. Conjunctival proinflammatory and proapoptotic effect of latanoprost and preserved and unpreserved timolol: an ex vivo and in vitro study. Inv Ophthalmol Vis Sci. 2004;45:1360-1368.

7. Guenoun JM, Baudouin C, Rat P, Pauly A, Warnet JM, Brignole-Baudouin F. In vitro study of inflammatory potential and toxicity profile of latanoprost, travoprost, and bimatprost in conjunctiva-derived epithelial cells. Inv Ophtalmol Vis Sci. 2005;46:2444-2450.

8. Guenoun J-M, Baudouin C, Rat P, Pauly A, Warnet J-M, Brignole-Baudouin F. In vitro comparison of cytoprotective and antioxidative effects of latanoprost, travoprost, and bimatopros on conjunctiva-derived epithelial cells. Inv Ophthalmol Vis Sci. 2005;46:4594-4599.

9. Pozarowska D, Pozarowski P, Darzynkiewicz Z. Cytometric assessment of cytostatic and cytotoxic effect of topical glaucoma medication on human epithelial corneal line cells. Cytometry Part B. 2010;78B:130-137.

10. Pozarowski P, Grabarek J, Darzynkiewicz Z. Flow cytometry of apoptosis. In: Robinson JP, Darzynkiewicz Z, Dean P et al. (eds). Current Protocols In Cytometry. John Wiley and Sons, (2003) 7.19.1-33.

11. Pozarowski P, Halicka DH, Darzynkiewicz Z. NF- $\kappa$ B inhibitor sesquiterpene parthenolide induces concurrently atypical apoptosis and necrosis: difficulties in identification of dead cells in such cultures. Cytometry A. 2003;54A:118-124.

12. Pozarowski P, Huang X, Halicka D, Lee B, Johnson G, Darzynkiewicz Z. Interaction of fluorochrome-labeled caspase inhibitors with apoptotic cells. A caution in data interpretation. Cytometry. 2003;55A:50-60.

13. Yee RW, Norcom EG, Zhao XC. Comparison of the relative toxicity of travoprost $0.004 \%$ without benzalkonium chloride and latanoprost $0.005 \%$ in an immortalized human corneal epithelial cell culture system. Adv Ther. 2006;23:511-519.

14. Baudouin C, Riancho L, Warnet JM, Brignole F. In vitro studies of antiglaucomatous prostaglandin analogues: travoprost with and without benzalkonium chloride and preserved latanoprost. Inv Ophtalmol Vis Sci. 2007;48:4123-4128.

15. Jaenen N, Baudouin C, Pouliquen P, Manni G, Figueiredo A, Zeyen T. Ocular symptoms and signs with preserved and preserve-free glaucoma medication. Eur J Ophthalmol. 2007;17:341-349. 
16. Brasnu E, Brignole-Baudouin F, Riancho L, Warnet JM, Baudouin C. Comparative study on the cytotoxic effect of benzalkonium chloride on the Wong-Kilboure derivative of Chang conjunctival and IOBA-NHC cell lines. Mol Vis. 2008;14:394-402.

17. Dutot M, Liang H, Pauloin T et al. Effects of toxic cellular stress and divalent caution on the human P2X7 cell death receptor. Mol Vis. 2008;14:889-897.

18. De Saint Jean M, Bringnole F, Bringuier AF et al. Effect of Benzalkonium Chloride on growth and survival of Chang conjunctival cells. Inv Ophthalmol Vis Sci. 1999;40:619-629.
19. De Saint Jean M, Debbasch C, Brignole F, Rat P, Warnet JM, Baudouin C. Toxicity of preserved and unpreserved antiglaucoma topical drugs in an in vitro model of conjunctival cells. Curr Eye Res. 2000;20:85-94.

20. Yu A, Fuchshofer R, Kampik A, Walge-Lussen U. Effects of oxidative stress in trabecular meshwork cells are reduced by prostaglandin analogues. Inv Ophthalmol Vis Sci. 2008; 11:4872-4880.

21. Wu KY, Wang HZ, Hong SJ. Effects of antiglaucoma drugs on cellular proliferation in cultured human corneal keratocytes. Kaohsiung J Med Sci. 2006;22:120-235.

Submitted: 29 June, 2010

Accepted after reviews: 30 November, 2010 